\title{
A Case Study on the Disease Burden and Influencing Factors of Imported Malaria Patients in a County-Level Hospital - Guangxi Zhuang Autonomous Region, China, 2016-2019
}

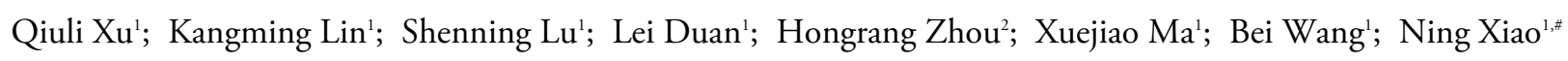

\section{Summary}

What is already known on this topic?

Imported malaria cases endanger people's health and potentially cause local re-transmission, and they may also cause economic loss on patients' families and society as a whole.

What is added by this report?

This is the first report to focus on the disease burden of a case study incurred by the imported malaria. The results indicated that the median direct medical cost was 2,904.4 CNY and the median indirect cost was 242.0 CNY for a patient's hospitalization. The economic cost was related to age, time between onset and diagnosis, and days of stay in hospital.

What are the implications for public health practice?

This study analyzed the main causes based on both direct and indirect economic loss of imported malaria cases to provide general information for the evaluation of the disease burden of imported malaria patients and shed light on the rational allocation of medical resources.

Although no locally transmitted malaria cases have been reported in China since 2017, the number of imported cases still remained high in recent years. From 2017 to 2019, China has reported 8,202 imported malaria cases and 33 deaths (1-4). Imported malaria not only puts the health and safety of local residents at risk, but also takes an economic toll on the patients' families and the communities (5). This paper assessed the disease burden of imported malaria patients and possible determinants based on economic loss, which aims to inform policymaking and promote rational allocation of medical resources in China during the elimination and post-elimination stage. The study targeted imported malaria patients who visited the People's Hospital of Shanglin County (a designated hospital for malaria diagnosis and treatment) in
Guangxi Zhuang Autonomous Region from January 1, 2016 to December 31, 2019 to calculate the economic cost during stay in the hospital and explore influencing factors through univariate analysis and multiple linear regression. The median of direct medical cost was 2,904.4 CNY and the median of indirect cost was 242.0 CNY. Univariate analysis showed that the direct cost was related to days of stay in hospital, and there was no significant difference in indirect cost among groups. Multiple linear regression analysis indicated total economic burden depended on the patients' age, interval between onset and diagnosis, and days of stay in hospital. Therefore, standardized treatment leading to normal hospital stay and enhanced health education on malaria prevention can effectively reduce the economic loss of imported malaria patients.

Guangxi Zhuang Autonomous Region is located in the south of China where malaria was historically highly prevalent. A total of 3,195 malaria cases were reported in Guangxi from 2010 to 2019, of which 3,193 were imported cases. Most imported malaria cases were $P$. falciparum infection, which accounted for $72.3 \%(2,310 / 3,193)$, followed by $P$. ovale $13.9 \%$ $(444 / 3,193)$. According to the report, the medical information of $3,159 / 3,193(98.94 \%)$ patients was obtained, in which $70.7 \%(2,233 / 3,159)$ were treated in Shanglin County medical institutions and the local CDC (6). Therefore, Shanglin County was selected as the study site.

The disease burden evaluation of imported malaria patients in this study was comprised of direct cost and indirect cost. Direct cost contains hospitalization expenses, while indirect cost refers to economic loss caused by work delays during the treatment. The formula for indirect cost in this study was as follows: indirect costs $=$ hospitalized days $\times$ Shanglin County GDP per person/365. Based on the Statistical Bulletin of National Economic and Social Development of Shanglin County in 2019, the GDP per capita of the county is 22,086 CNY, and the average daily GDP per 
capita is 60.5 CNY (22,086.0/365).

In this study, the People's Hospital of Shanglin was selected as the target institution. The case information of all imported malaria patients treated in the hospital from January 1, 2016 to December 31, 2019 was collected through medical records investigation for further analysis, excluding data with logic errors and incomplete information. Considering the impact of price growth and other factors on hospitalization costs in different years, the hospitalization costs from 2016 to 2018 were standardized by consulting the Consumer Price Index (CPI) of Guangxi from 2017 to 2019 (7-9) and taking the hospitalization costs in 2019 as the baseline. For example, hospitalization expenses in 2016 (standardized) $=$ hospitalization expenses in $2016 \times(1+2017$ 's CPI $) \times(1+2018$ 's CPI $) \times(1+2019$ 's CPI $)$. The case information included the patient's gender, age, infected Plasmodium species, interval between onset and diagnosis, days of stay in hospital, hospitalization cost, and the cost of each item, etc.

The data were recorded in Excel and statistical analyses were performed using SAS software (version 9.4, SAS Institute, Cary, NC, USA). Descriptive statistical analysis was used to describe the general characteristics of the patients. Since direct costs, indirect costs, and total costs did not meet the normal distribution, the quantitative data were analyzed by means, median, and Q1 and Q3. Wilcoxon rank sum test was carried out for comparison between two groups, and Kruskal-Wallis one-way ANOVA test was conducted for comparison between multiple groups. Through square root transformation, the total economic burden satisfied the normal distribution, and multiple linear regression analysis was performed stepby-step to screen independent variables using test level $\alpha=0.05$.

The results showed that 537 imported malaria cases were collected from the People's Hospital of Shanglin, including 527 males and 10 females. The median age was 40 years, with patients aged 20-49 years accounting for $77.1 \%$ (414/537), and each patient was hospitalized for 4 days. The median direct cost was $2,904.4 \mathrm{CNY}$, accounting for $92.3 \%$ of the total expenditures $(2,904.4 / 3,146.4)$, with the cost of laboratory tests, drug products, and examination as the top three costs. The median indirect cost was 242.0 CNY, accounting for $7.7 \%$ of the total economic burden $(242.0 / 3,146.4)$ (Figure 1). Univariate analysis showed that direct expenses were only related to days of stay in the hospital and that the direct cost of

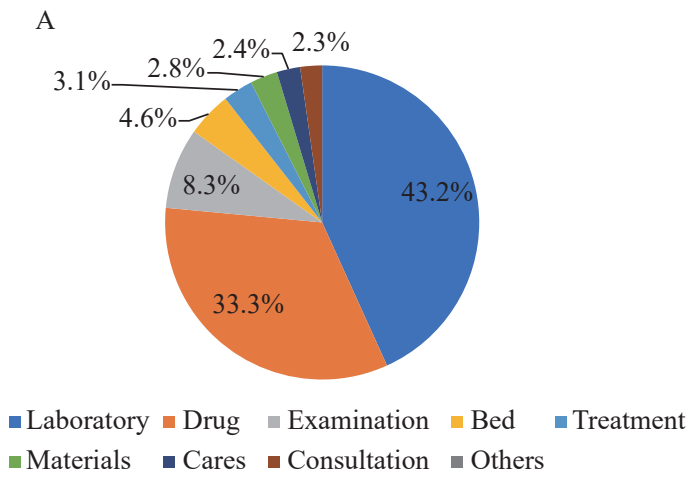

B

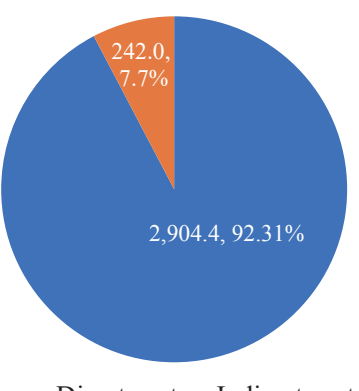

FIGURE 1. Composition of economic costs of imported malaria patients in Shanglin County, Guangxi Zhuang Autonomous Region from 2016 to 2019. (A) Proportion of direct costs of imported malaria patients. (B) Proportion of the total economic burden of imported malaria patients.

hospital stay $\geq 4$ days group was higher than that of hospital stay $<4$ days group (Table 1 ). There was no significant difference in indirect cost among the groups (Table 1). Multiple linear regression analysis of the total economic burden showed that total cost was highly related to age, days of stay in hospital, and onset-diagnosis time (Table 2).

\section{DISCUSSION}

The total economic burden of a malaria case was about 3,146.4 CNY, which was $14.2 \%$ of the local per capita GDP, bringing a major economic burden to families of the patients. Most malaria inpatients in the People's Hospital of Shanglin were males aged 20-49 years, which was consistent with the characteristics of most imported malaria cases being migrant workers (10). Among the direct costs of malaria-related treatment, the costs of laboratories, drugs, and examinations were relatively high, which may be caused by the frequent examination of blood routine, blood biochemistry, urine routine, and other indicators for malaria diagnosis, disease progress, and drug side effects. Although antimalarial drugs were free of 
TABLE 1. The average medical costs of imported malaria patients in Shanglin County, Guangxi Zhuang Autonomous Region, 2016-2019.

\begin{tabular}{|c|c|c|c|c|c|c|c|c|c|c|c|}
\hline \multirow{2}{*}{ Indicator } & \multirow{2}{*}{ Total, $\mathrm{n}(\%)$} & \multicolumn{5}{|c|}{ Direct cost } & \multicolumn{5}{|c|}{ Indirect cost } \\
\hline & & Mean & Median & $(Q 1, Q 3)^{*}$ & $\mathrm{Z} / \mathrm{X}^{2}$ value & $P$ value & Mean & Median & $(Q 1, Q 3)^{*}$ & $\mathrm{Z} / \mathrm{X}^{2}$ value & $P$ value \\
\hline \multicolumn{12}{|l|}{ Gender } \\
\hline Female & $527(98.1)$ & $3,030.8$ & $2,895.4$ & $(2,408.9,3,459.2)$ & 1.11 & 0.26 & 244.6 & 242.0 & $(181.5,302.5)$ & 1.2 & 0.23 \\
\hline Male & $10(1.9)$ & $3,178.1$ & $3,309.0$ & $(2,748.9,3,560.6)$ & & & 260.2 & 242.0 & $(242.0,302.5)$ & & \\
\hline Age (years) & & & & & 4.29 & 0.12 & & & & 3.2 & 0.20 \\
\hline$<20$ & $2(0.4)$ & $2,576.2$ & $2,576.2$ & $(2,428.8,2,723.6)$ & & & 211.8 & 211.8 & $(181.5,242.0)$ & & \\
\hline $20-49$ & $414(77.1)$ & $3,014.9$ & $2,861.0$ & $(2,414.3,3,444.6)$ & & & 242.7 & 242.0 & $(181.5,302.5)$ & & \\
\hline$\geq 50$ & $121(22.5)$ & $3,104.7$ & $3,056.7$ & $(2,407.6,3,556.8)$ & & & 253.0 & 242.0 & $(181.5,302.5)$ & & \\
\hline \multicolumn{2}{|c|}{ Onset-diagnosis days (days) } & & & & 0.80 & 0.42 & & & & 1.4 & 0.18 \\
\hline$\leq 1$ & $289(53.8)$ & $2,993.6$ & $2,895.4$ & $(2,433.9,3,284.1)$ & & & 237.2 & 242.0 & $(181.5,242.0)$ & & \\
\hline$>1$ & $248(46.2)$ & $3,080.0$ & $2,935.9$ & $(2,390.6,3,524.1)$ & & & 254.0 & 242.0 & $(181.5,302.5)$ & & \\
\hline \multicolumn{2}{|c|}{ Length of stay (days) } & & & & -10.07 & $<0.01$ & & & & - & - \\
\hline$<4$ & $186(34.6)$ & $2,540.9$ & $2,559.5$ & $(2,060.0,2,874.7)$ & & & - & - & - & & \\
\hline$\geq 4$ & $351(65.4)$ & $3,294.5$ & $3,129.9$ & $(2,693.4,3,690.9)$ & & & - & - & - & & \\
\hline \multicolumn{2}{|c|}{ Species classification } & & & & 2.16 & 0.71 & & & & 3.7 & 0.45 \\
\hline P. falciparum & $328(61.1)$ & $3,101.2$ & $2,916.2$ & $(2,433.9,3,494.0)$ & & & 252.0 & 242.0 & $(181.5,302.5)$ & & \\
\hline P. ovale & $176(32.8)$ & $2,931.5$ & $2,920.3$ & $(2,380.7,3,443.3)$ & & & 232.4 & 242.0 & $(181.5,302.5)$ & & \\
\hline P. vivax & $17(3.2)$ & $2,800.0$ & $2,713.8$ & $(2,219.6,3,462.6)$ & & & 249.1 & 242.0 & $(181.5,302.5)$ & & \\
\hline Mixed-infection & $9(1.7)$ & $3,081.1$ & 2,895.4 & $(2,754.1,3,500.2)$ & & & 235.3 & 242.0 & $(181.5,242.0)$ & & \\
\hline P. malariae & $7(1.3)$ & $2,930.8$ & $3,140.1$ & $(2,802.6,3,245.0)$ & & & 233.4 & 242.0 & $(242.0,242.0)$ & & \\
\hline
\end{tabular}

Q1, known as the "Smaller Quartile", is equal to the 25\% of all values in the sample, from smallest to largest. Q3, known as the "higher fourth quantile", is equal to the $75 \%$ of all values in the sample, from smallest to largest.

TABLE 2. Multiple linear regression results of total cost of imported malaria patients in Shanglin County, Guangxi Zhuang Autonomoums Region, 2016-2019.

\begin{tabular}{|c|c|c|c|c|c|}
\hline Variable & $\beta$ & Standard error & Standard $\beta$ & $t$ value & $P$ value \\
\hline Intercept & $1,822.8$ & 151.3 & 0 & 12.1 & $<0.01$ \\
\hline Age & 7.7 & 3.3 & 0.1 & 2.4 & 0.02 \\
\hline Length of stay & 268.6 & 17.7 & 0.5 & 15.1 & $<0.01$ \\
\hline Onset-diagnosis interval (days) & -3.3 & 1.5 & -0.1 & -2.2 & 0.03 \\
\hline
\end{tabular}

charge, other drugs for related symptoms and supportive treatment drugs were required during hospitalization. The differential diagnosis of malaria and other diseases often required B-scan ultrasound, electrocardiogram, $\mathrm{X}$ ray and other examinations.

Univariate analysis showed that longer stays in the hospital affected the direct cost of hospital stay as the hospital stay $\geq 4$ days group had higher costs than the hospital stay $<4$ days group. The differences in indirect costs between groups were not statistically significant, which means that the differences in hospitalization days between groups were not statistically significant. For univariate analysis, both age and onset-diagnosis interval were included as categorical variables, while continuous variables were included for multiple linear regression, which could explain the difference in results. Multiple linear regression analysis showed that age, longer stay, and the onset-diagnosis interval affected the total cost. The interval between onset and diagnosis might affect the severity of the patient's illness. The longer the time spent from onset to treatment for diagnosis, the worse the patient will become, leading to an increase in economic costs. The length of the hospital stay might be related to the severity of the patient's symptoms and various indicators of the body of patients. As the hospital stay was extended, the total costs of various items including bed fee, medical consultation fee, and nursing fee 
would rise accordingly, thus causing higher hospital costs.

This study was subject to some limitations. First, the direct economic burden included direct medical expenses and direct non-medical expenses. Because imported malaria cases were scattered and there were little direct non-medical burden data available through the field investigations, the study did not include direct non-medical expenses, such as meals, accommodation, and transportation for patients and their families. Second, it was found in a survey in Shanglin County that only $6.7 \%(2 / 30)$ of the imported malaria patients had a caretaker to take care of them all day in the hospital. Therefore, the indirect economic burden in this study only took into account for patients who missed work due to illness. Finally, the relatively poor balance between groups contributed to poor comparability between groups, such as gender grouping.

In conclusion, the economic burden of imported malaria patients in Guangxi Zhuang Autonomous Region was affected by age, the length of hospitalization, and the interval between onset and diagnosis. Therefore, by advocating reasonable medical treatment behavior and standardizing hospitalization, discharge, and the length of hospitalization, the treatment costs of imported malaria patients could be effectively reduced. In addition, one of the most crucial strategies and approaches to reducing the malaria disease burden is to strengthen health education and personal self-protection for people who travel abroad to endemic areas so as to reduce the chance of malaria infection, mitigate the threat of malaria importation, and consolidate the achievements of malaria elimination.

Funding: Supported by the National Key Research and Development Program of China (No. 2016YFC1200502) and the WHO Biennial Cooperative Project (No. 2012/269948-0).

doi: $10.46234 / \mathrm{ccdcw} 2021.096$
\# Corresponding author: Ning Xiao, xiaoning@nipd.chinacdc.cn.

\begin{abstract}
${ }^{1}$ National Institute of Parasitic Diseases, Chinese Center for Disease Control and Prevention; Chinese Center for Tropical Diseases Research; WHO Collaborating Centre for Tropical Diseases; National Center for International Research on Tropical Diseases, Ministry of Science and Technology; Key Laboratory of Parasite and Vector Biology, Ministry of Health, Shanghai, China; ${ }^{2}$ Qingpu District Center for Disease Control and Prevention, Shanghai, China.
\end{abstract}

Submitted: February 26, 2021; Accepted: April 21, 2021

\section{REFERENCES}

1. Zhang L, Feng J, Zhang SS, Xia ZG, Zhou SS. The progress of national malaria elimination and epidemiological characteristics of malaria in China in 2017. Chin J Parasitol Parasit Dis 2018;36(3):201-9. http://www.jsczz.cn/CN/Y2018/V36/I3/2. (In Chinese).

2. Zhang L, Feng J, Zhang SS, Xia ZG, Zhou SS. Epidemiological characteristics of malaria and the progress towards its elimination in China in 2018. Chin J Parasitol Parasit Dis 2019;37(3):241 - 7. http://dx.doi.org/10.12140/j.issn.1000-7423.2019.03.001. (In Chinese).

3. Zhang L, Feng J, Xia ZG, Zhou SS. Epidemiological characteristics of malaria and progress on its elimination in China in 2019. Chin J Parasitol Parasit Dis 2020;38(2):133 - 8. http://dx.doi.org/10.12140/ j.issn.1000-7423.2020.02.001. (In Chinese).

4. Xiao N, Xu QL, Feng J, Xia ZG, Duan L, Wang DQ, et al. Approaching malaria elimination in China. China CDC Wkly 2020;2(17):293 - 7. http://dx.doi.org/10.46234/ccdcw2020.075.

5. Xu QL, Zhou HR, Qian MB, Wang DQ, Xiao N. Research progress on economic burden of malaria. Chin J Parasitol Parasit Dis 2020; 38(6):749 - 52. http://dx.doi.org/10.12140/j.issn.1000-7423.2020.06. 012. (In Chinese).

6. Li J, Wei SJ, Zhang WW, Lin KM, Yan H, Feng XY. Analysis of malaria epidemiological characteristics in Guangxi in 2010-2019. Chin J Parasitol Parasit Dis 2020;38(5):589 - 94. http://dx.doi.org/10. 12140/j.issn.1000-7423.2020.05.010. (In Chinese).

7. Guangxi Zhuang Autonomous Region Bureau of Statistics. Statistical bulletin of national economic and social development of Guangxi Zhuang autonomous region, 2017. http://tjj.gxzf.gov.cn/tjsj/tjgb/ ndgmjjhshfz/t2382810.shtml. [2018-4-16]. (In Chinese).

8. Guangxi Zhuang Autonomous Region Bureau of Statistics. Statistical bulletin of national economic and social development of Guangxi Zhuang autonomous region, 2018. http://tjj.gxzf.gov.qqgb/t2381649. shtml. [2019-4-10]. (In Chinese).

9. Guangxi Zhuang Autonomous Region Bureau of Statistics. Statistical bulletin of national economic and social development of Guangxi Zhuang autonomous region, 2019. http://tjj.gxzf.gov.cn/tjsj/tjgb/ ndgmjjhshfz/t2382864.shtml. [2020-3-17]. (In Chinese).

10. Liu YB, Hsiang MS, Zhou HY, Wang WM, Cao YY, Gosling RD, et al. Malaria in overseas labourers returning to China: an analysis of imported malaria in Jiangsu Province, 2001-2011. Malar J 2014;13:29. http://dx.doi.org/10.1186/1475-2875-13-29. 